University of New Orleans

ScholarWorks@UNO

10-1972

\title{
Analogy Between Linear Optical Systems and Linear Two-Port Electrical Networks
}

\author{
Rasheed M.A. Azzam \\ University of New Orleans, razzam@uno.edu
}

N. M. Bashara

Follow this and additional works at: https://scholarworks.uno.edu/ee_facpubs

Part of the Electrical and Electronics Commons

\section{Recommended Citation}

R. M. A. Azzam and N. M. Bashara, "Analogy Between Linear Optical Systems and Linear Two-Port Electrical Networks," Appl. Opt. 11, 2210-2214 (1972) http://www.opticsinfobase.org/ao/ abstract.cfm?URI=ao-11-10-2210.

This Article is brought to you for free and open access by the Department of Electrical Engineering at ScholarWorks@UNO. It has been accepted for inclusion in Electrical Engineering Faculty Publications by an authorized administrator of ScholarWorks@UNO. For more information, please contact scholarworks@uno.edu. 


\title{
Analogy Between Linear Optical Systems and Linear Two-Port Electrical Networks
}

\author{
R. M. A. Azzam and N. M. Bashara
}

\begin{abstract}
Attention is called to the analogy between linear optical systems and linear two-port electrical networks. For both, the transformation of a pair of oscillating quantities between input and output is of interest. The mapping of polarization by an optical system and of impedance (admittance) by a two-port network is described by a bilinear transformation. Therefore for each transfer property of a system of one type, there is a similar property for the system of the other type. Two-port electrical networks are synthesized whose impedance-(or admittance-) mapping properties are the same as the polarization-mapping properties of a given optical system. The opposite problem of finding the optical analogs of two-port networks is also considered. Besides unifying the methods of handling these two different kinds of systems, the analogy appears fruitful if used reciprocally to simulate electrical networks by optical systems, and vice versa. Linear mechanoacoustic systems have optical analogs besides their well-known electrical analogs.
\end{abstract}

\section{Introduction}

The advent of microwaves and more recently of lasers has stimulated the interest of electrical engineers in optics and optical systems. Establishing analogies between electrical networks and optical systems would be useful in (1) unifying the methods of treating both kinds of systems and (2) the reciprocal simulation of systems of one type by systems of the other.

\section{Basis of the Analogy}

The basis of the analogy between optical systems and two-port electrical networks ${ }^{1}$ is explained with reference to Fig. 1. For the optical system $S$, shown in Fig. 1(a), nonlinear optical effects and other frequency-changing phenomena (such as Brillouin or Raman scattering) are assumed absent. Also, incoherent scattering processes are excluded. Under these conditions the incident and

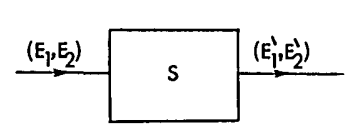

(a)

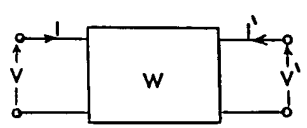

(b)
Fig. 1. (a) Linear optical system $S$; (b) linear two-port network $W$. For $S$ and $W$ the transformation of a pair of oscillating quantities between input and output is of interest.

The authors are with the Electrical Materials Laboratory, Department of Electrical Engineering, University of Nebraska, Lincoln, Nebraska 68508.

Received 13 March 1972. outgoing waves are monochromatic and of the same frequency, and both are totally polarized. The polarization states of these waves can be specified with reference to any chosen pair of basis states, which are generally elliptic. Any polarization state can be generated by a linear superposition of the basis states 1 and 2 using complex coefficients $E_{1}$ and $E_{2}$, respectively. These coefficients transform linearly after propagation through the system $S$

$$
\left(\begin{array}{c}
E_{1}{ }^{\prime} \\
E_{2}{ }^{\prime}
\end{array}\right)=\left(\begin{array}{ll}
T_{11} & T_{12} \\
T_{21} & T_{22}
\end{array}\right)\left(\begin{array}{l}
E_{1} \\
E_{2}
\end{array}\right),
$$

where $T_{i j}$ denote the complex elements of the generalized Jones matrix ${ }^{2}$ of the optical system. The matrix $T$ depends both on the internal structure of $S$ and on the basis states used at the input and output of the optical system, which need not be the same. When two orthogonal linear polarizations are chosen as basis states, Eq. (1) becomes

$$
\left(\begin{array}{c}
E_{x}{ }^{\prime} \\
E_{y}{ }^{\prime}
\end{array}\right)=\left(\begin{array}{cc}
T_{11}^{x y} & T_{12}^{x y} \\
T_{21}^{x y} & : T_{22}^{x y}
\end{array}\right)\left(\begin{array}{c}
E_{x} \\
E_{y}
\end{array}\right),
$$

where $\left(T_{i i}^{x y}\right)$ refers to the ordinary cartesian Jones matrix and the elements of the vector $\mathbf{E}$ correspond to the cartesian components of the electric vector of the light wave.

In many cases we are interested in the polarizationmapping properties of the system $S$ overlooking any over-all amplitude or phase changes between input and . output. The polarization forms (or the ellipses of polarization) of the incident and outgoing waves are determined by the complex ratios $\chi$ and $\chi^{\prime}$, respectively, where 


$$
\chi=E_{2} / E_{1}, \quad \chi^{\prime}=E_{2}{ }^{\prime} / E_{1}{ }^{\prime} .
$$

According to Eq. (1) $\chi^{\prime}$ and $\chi$ are interrelated by

$$
\chi^{\prime}=\left(T_{22} \chi+T_{21}\right) /\left(T_{12} \chi+T_{11}\right),
$$

which is a bilinear transformation. The result in Eq. (4) has been utilized ${ }^{3-6}$ to investigate various aspects of polarization transfer by optical systems and to provide a procedure ${ }^{5}$ (generalized ellipsometry) to measure the matrix $T$.

The two-port network shown in Fig: 1(b) is assumed linear and may contain active elements operating in the linear domain. ${ }^{7}$ The voltage and current $V^{\prime}$ and $I^{\prime}$ at one port are related to the voltage and current $V$ and $I$ at the other port by a linear transformation

$$
\left(\begin{array}{l}
I^{\prime} \\
V^{\prime}
\end{array}\right)=\left(\begin{array}{ll}
W_{11} & W_{12} \\
W_{21} & W_{22}
\end{array}\right)\left(\begin{array}{l}
I \\
V
\end{array}\right),
$$

where $W_{i j}$ are the complex elements of the network transfer matrix. ${ }^{8}$ The impedances at the two ports are defined by

$$
Z=V / I, \quad Z^{\prime}=V^{\prime} / I^{\prime},
$$

and according to Eq. (5) they are related by

$$
Z^{\prime}=\left(W_{22} Z+W_{21}\right) /\left(W_{12} Z+W_{11}\right),
$$

which is a bilinear transformation. Obviously, the transformation of admittance between the two ports is also bilinear,

$$
Y^{\prime}=\left(W_{11} Y+W_{12}\right) /\left(W_{21} Y+W_{22}\right)
$$

The analogy between optical systems and two-port networks is clearly demonstrated by the similarity of Eqs. (1) and (5) and Eqs. (4) and (7) or (8). In both cases we deal with the transformation of (the complex amplitudes of) a pair of oscillating quantities between the input and output. These are the two oscillating components of the light vector along the two chosen basis states in the case of optical systems and the voltage and current oscillations in the case of two-port networks.

Rumsey has previously shown the similarity of the definition of impedance and polarization ratios and suggested the use of impedance charts (Smith or Carter) to represent elliptical polarizations. ${ }^{9}$ Deschamps briefly mentioned the similarity between the mapping of polarization by optical systems and of impedance by electrical networks. ${ }^{10}$

\section{Analogous Properties of Optical Systems and Two-Port Networks}

From the previous section we have seen that the mapping of polarization by an optical system and of impedance (or admittance) by a two-port electrical network between input and output is described by a bilinear transformation. It follows that for each terminal characteristic of a system of one type there is a similar characteristic for the system of the other type.

First we note that a bilinear transformation can always be found that maps any three points $\left(\zeta_{1}, \zeta_{2}, \zeta_{3}\right)$ in the complex $\zeta$ plane into the three points $\left(\eta_{1}, \eta_{2}, \eta_{3}\right)$ in the complex $\eta$ plane and is given $b y^{3,11}$

$$
\begin{array}{r}
\left(\eta-\eta_{1}\right) /\left(\eta-\eta_{2}\right)=\left[\left(\eta_{3}-\eta_{1}\right)\left(\zeta_{3}-\zeta_{2}\right) /\left(\eta_{3}-\eta_{2}\right)\left(\zeta_{3}-\zeta_{1}\right)\right] \\
\times\left[\left(\zeta-\zeta_{1}\right) /\left(\zeta-\zeta_{2}\right)\right]
\end{array}
$$

This means that if the transformation of three polarization states by an optical system $S$ or three impedances by a network $W$ is known, the mapping of all other polarizations or impedances is completely determined: The bilinear transformation is referred to as the polarization transfer function (PTF) or the impedance transfer function (ITF). According to Eqs. (4) and (7), the coefficients of the PTF and the ITF determine the matrices $T$ and $W$ of Eqs. (1) and (5), respectively, up to a complex multiplier. Besides the mapping of three polarizations or three impedances, a fourth measurement is needed to determine these matrices completely. This involves the determination of the over-all phase delay and attenuation of a light wave of a given polarization by the optical system and a complex voltage- or current-transfer ratio for the network.

For any choice of the basis states Eq. (3) leads to a complex-plane representation in which the loci of elliptic polarizations of the same azimuth and of the same ellipticity constitute two orthogonal sets of circles. These equiazimuth and equiellipticity families of circles respectively pass through and enclose the two points in the complex plane that represent the right and left circular polarizations. If the latter polarizations are chosen as the basis states, the equiazimuth and equiellipticity contours become straight lines and concentric circles through and around the origin, respectively. In the impedance plane these straight lines and circles through and around the origin correspond to the loci of impedances of constant angle and of constant magnitude, respectively. The origin and the point at infinity correspond to the left and right circular polarizations in one case and to zero impedance (short circuit) and infinite impedance (open circuit) in the other.

An important property of bilinear transformations between two complex planes is that a circle in one plane is mapped into a circle in the other plane. Applying this property, it is seen that if an incident polarization $\chi$ is of a fixed azimuth (ellipticity), the polarization $\chi^{\prime}$ exiting from the optical system $S$ will traverse a circle in the complex plane as the ellipticity (azimuth) of $\chi$ is varied to scan all possible values. ${ }^{3}$ Similarly, for the two-port network, $W$, if an impedance $Z$ of constant angle (magnitude) is connected at one port of the impedance, $Z^{\prime}$ that appears at the other port will follow a circle in the complex plane as the magnitude (angle) of $Z$ is varied to cover all possible values. The optical system $S$ and the two-port network $W$ map the two orthogonal families of straight lines and concentric circles through and around the origin in the polarization-impedance $(\chi-Z)$ plane into two orthogonal sets of circles in the $\left(\chi^{\prime}-Z^{\prime}\right)$ plane (Fig. 2$)$. The preservation of orthogonality is a consequence of the conformal nature of the transformation. The two points $A^{\prime}$ and $B^{\prime}$ in the $\left(\chi^{\prime}-Z^{\prime}\right)$ plane are the images of the origin $A$ and the point at infinity $B$ of the $(\chi-Z)$ plane. $A^{\prime}$ and $B^{\prime}$ represent the response of $S$ to incident left and right circular polarizations and they also represent the impe- 

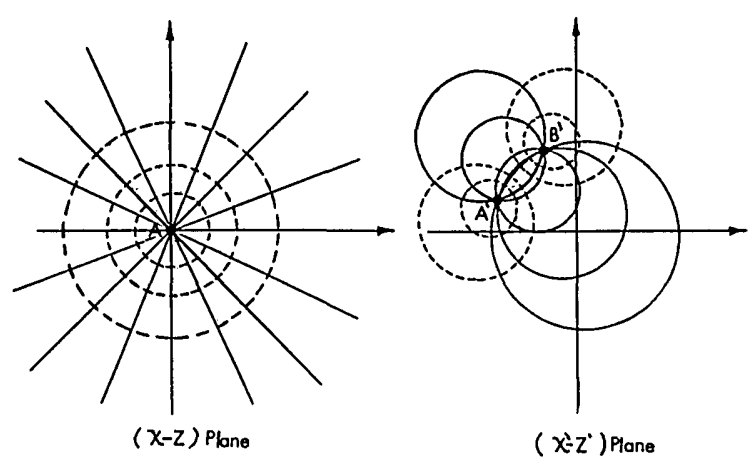

Fig. 2. The two orthogonal families of straight lines (-) and concentric circles (-) through and around the origin in the polarization-impedance $(\chi-Z)$ plane (left) represent polarization states of equiazimuth and equiellipticity and impedances of constant angle and constant magnitude, respectively. These are mapped by the optical system $S$ and the two-port netowrk $W$ into the orthogonal families of circles passing through and enclosing the two points $A^{\prime}$ and $B^{\prime}$ (right). $A^{\prime}$ and $B^{\prime}$ in the $\left(\chi^{\prime}-\right.$ $Z^{\prime}$ ) plane are the images of the origin $(A)$ and the point at infinity $(B)$ of the $(\chi-Z)$ plane.

dance that appears at one part of $W$ when the other port is short- and open-circuited, respectively.

For each optical system there are two polarization states that pass through the system unchanged. These eigenpolarizations ${ }^{3}$ are obtained from Eq. (4) by setting $\chi^{\prime}=\chi$. This yields

$\chi_{e 1,2}=\left(1 / 2 T_{12}\right)$

$$
\times\left\{\left(T_{22}-T_{11}\right) \pm\left[\left(T_{22}-T_{11}\right)^{2}+4 T_{12} T_{21}\right]^{\frac{1}{2}}\right\} .
$$

Corresponding to the eigenpolarizations $\chi_{e 1}$ and $\chi_{e 2}$ of the optical system $S$, the two-port network $W$ has two iterative impedances $Z_{i 1}$ and $Z_{i 2}$ that when connected at one port appear unchanged at the other. By analogy, Eq. (7) gives

$$
\begin{aligned}
Z_{i 1,2}= & \left(1 / 2 W_{12}\right) \\
& \times\left\{\left(W_{22}-W_{11}\right) \pm\left[\left(W_{22}-W_{11}\right)^{2}+4 W_{12} W_{21}\right]^{\frac{1}{2}}\right\} .
\end{aligned}
$$

Because Eqs. (10) and (11) involve ratios of the elements of the transfer matrices $T$ and $W$, the eigenpolarizations and iterative impedances are uniquely determined by the PTF and ITF, respectively.

The loci of polarization states that preserve either ellipticity or azimuth after propagation through an optical system ${ }^{6}$ are analogous to the loci of impedances that if connected at one port would appear at the other with either magnitude or angle unchanged, respectively. To determine the cartesian equation of these loci, both Eqs. (4) and (7) are put in the form

$$
\eta=(A \zeta+B) /(C \zeta+D),
$$

where $\zeta=\chi$ or $Z ; \eta=\chi^{\prime}$ or $Z^{\prime}$, and the coefficients $A$, $B, C$, and $D$ correspond to the elements of either the $T$ matrix of the optical system or the $W$ matrix of the two-port network. The locus of polarizations that preserve their ellipticity or impedances that preserve their magnitude is determined by ${ }^{12}$

$$
|\eta|=|\xi|
$$

or

$$
|(A \zeta+B) /(C \zeta+D)|=|\zeta| .
$$

Similarly the locus of polarizations that preserve their azimuth or impedances that preserve their angle is given by ${ }^{12}$

$$
\operatorname{Arg}(\eta)=\operatorname{Arg}(\zeta)
$$

or

$$
\operatorname{Arg}[(A \zeta+B) /(C \zeta+D)]=\operatorname{Arg}(\zeta) .
$$

Expanding Eqs. (14) and (16) yields

$$
\left(x^{2}+y^{2}\right) Q_{1}-Q_{2}=0,
$$

and

$$
x Q_{3}-y Q_{4}=0,
$$

respectively. In Eqs. (17) and (18) we have

$$
\zeta=x+j y
$$

and the $Q$ 's are quadratics in $x$ and $y$ given by

$$
\begin{aligned}
& Q_{1}=(\mathbf{C} \cdot \mathbf{C})\left(x^{2}+y^{2}\right)+(\mathbf{C} \cdot \mathbf{D}) x+2(\mathbf{C} \times \mathbf{D}) y+(\mathbf{D} \cdot \mathbf{D}), \\
& Q_{2}=(\mathbf{A} \cdot \mathbf{A})\left(x^{2}+y^{2}\right)+2(\mathbf{A} \cdot \mathbf{B}) x+2(\mathbf{A} \times \mathbf{B}) y+(\mathbf{B} \cdot \mathbf{B}), \\
& Q_{3}=(\mathbf{C} \times \mathbf{A})\left(x^{2}+y^{2}\right)-(\mathbf{B} \times \mathbf{C}-\mathbf{D} \times \mathbf{A}) x \\
& -(\mathbf{B} \cdot \mathbf{C}-\mathbf{D} \cdot \mathbf{A}) y+(\mathbf{D} \times \mathbf{B}) \text {, } \\
& Q_{4}=(\mathbf{C} \cdot \mathbf{A})\left(x^{2}+y^{2}\right)+(\mathbf{B} \cdot \mathbf{C}+\mathbf{D} \cdot \mathbf{A}) x \\
& -(\mathbf{B} \times \mathbf{C}+\mathbf{D} \times \mathbf{A}) y+(\mathbf{D} \cdot \mathbf{B}) .
\end{aligned}
$$

In Eq. (20) A, B, C , and $\mathbf{D}$ are the complex coefficients of the PTF or the ITF considered as vectors in the complex plane. The operations of the dot and cross products have their usual meanings except that for the cross product only the magnitude (with proper sign) is to be taken. Equations (17)-(20) can be easily used to determine these loci for any optical system or two-port network. The explicit form of the equations of the loci of invariant-ellipticity states and invariant-azimuth states of an optical system in the cartesian complexplane representation can be found in Ref. 6 .

\section{Simulation of Optical Systems by Equivalent Two-Port Networks and Vice Versa}

Because of the analogy between the terminal characteristicsiof optical systems and two-port networks, it is possible to simulate systems of one type by systems of the other. The equivalence is achieved when the PTF of the optical system [Eq. (4)] and the ITF (or the admittance transfer function) of the two-port network [Eq. (7) or (8)] are identical.

For a given optical system (two-port network) there is one and only one PTF (ITF). However, the opposite is not true, namely, that there can be many optical systems (two-port networks), all of which have the same PTF (ITF). In other words, there can be many possibilities for the internal structure of $S$ or $W$, all of which lead to the same terminal polarization- or impedance-mapping properties. 
Consider the following problem. Given an optical system $S$, find a two-port network $W$ whose impedanceor admittance-transforming properties are the same as the polarization-mapping properties of $S$. The first step in the solution is to find the PTF of $S$. As is evident from Eq. (4), the Jones matrix $T$ has to be determined apart from a complex multiplying factor. Because $T$ depends on the internal structure of $S$ as well as the basis polarization states, the latter have to be chosen. Such a choice is determined by the system under consideration and in most cases it is between a pair of orthogonal linear polarizations, the right and left circular polarizations or a mixture of these. Once the basis states have been decided upon, the Jones matrix $T$ is obtained from

$$
T=T_{N} \ldots T_{2} T_{1}
$$

where $T_{1}, T_{2} \ldots$, and $T_{N}$ are the matrices of the individual devices encountered by the light beam, with $T_{1}$ referring to the first-to-be-encountered optical element. Substituting the elements of the matrix $T$ of Eq. (21) into Eq. (4) the PTF of the optical system is found. The ITF or the admittance transfer function of an equivalent two-port network $W$ is obtained from Eq. (4) simply by substituting $Z$ or $Y$ for $\chi$ and $Z^{\prime}$ or $Y^{\prime}$ for $\chi^{\prime}$. This gives

$$
Z^{\prime}=\left(T_{22} Z+T_{21}\right) /\left(T_{12} Z+T_{11}\right)
$$

or

$$
Y^{\prime}=\left(T_{22} Y+T_{21}\right) /\left(T_{12} Y+T_{11}\right) .
$$

Thus the equivalence is between the polarization-mapping properties of $S$ and either the impedance- or the admittance-mapping properties of $W$. To synthesize a two-port network that maps an impedance $Z$ connected at one port into an impedance $Z^{\prime}$ at the other port in accordance with Eq. (22) we can rewrite the latter equation in the form

$$
Z^{\prime}=Z_{3}+\left[Z_{2}^{2} /\left(Z+Z_{1}\right)\right],
$$

where

$$
Z_{1}=T_{11} / T_{12}, \quad Z_{2}=(\operatorname{det} T)^{\frac{1}{2}} / T_{12}, \quad Z_{3}=T_{22} / T_{12},
$$

and $\operatorname{det} T$ denotes the determinant of the bilinear transformation,

$$
\operatorname{det} T=T_{11} T_{22}-T_{12} T_{21} .
$$

Equation (24) leads to the network of Fig. 3(a), which shows two series impedances $Z_{1}$ and $Z_{3}$ with a $\lambda / 4$ sec-

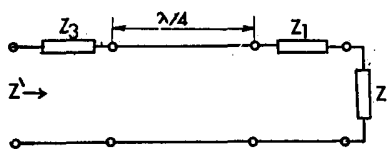

(a)

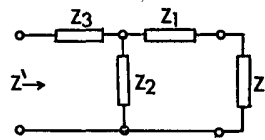

(b)
Fig. 3. Two-port electric networks with impedance-mapping properties identical to the polarization-mapping properties of a given optical system. The parameters of the networks (a) and (b) are given by Eqs. (25) and (27), respectively. tion of a transmission line of characteristic impedance $Z_{2}$ between them. A simpler (and more practical) synthesis is the $T$ network of Fig. 3(b) whose impedances $Z_{1}, Z_{2}$, and $Z_{3}$ are given by

$$
\begin{aligned}
Z_{1}=\left(T_{11} / T_{12}\right)-Z_{2}, \quad Z_{2}=(\operatorname{det} T)^{\frac{1}{2}} / T_{12}, \\
Z_{3}=\left(T_{22} / T_{12}\right)-Z_{2} .
\end{aligned}
$$

The corresponding alternative solutions based on the mapping of admittance instead of impedance between the two ports according to Eq. (23) are the duals of those shown in Fig. 3. Other network configurations with three independent impedances or admittances can be found that satisfy either Eq. (22) or Eq. (23).

The opposite problem is to find the optical analog $S$ of a given two-port network $W$. A direct procedure would be to search for an optical system $S$ with six real independent parameters (e.g., two dichroic retarders and a rotater) whose PTF is the same as the ITF or the admittance transfer function of $W$. In general the synthesis is not as straightforward as in the first case, when $S$ is given and $W$ is to be synthesized.

If $W_{1}, W_{2}, \ldots, W_{n}$ represent the two-port-network analogs of the optical systems $S_{1}, S_{2}, \ldots, S_{n}$, then the network formed by connecting $W_{1}, W_{2}, \ldots$. , and $W_{n}$ in succession will correspond to the cascade of the optical systems $S_{1}, S_{2}, \ldots$, and $S_{n}$ placed one after the other along the direction of propagation and in the same order in which the networks are connected.

It is interesting to find the electrical analogs of some of the simple optical elements. A polarizer is by definition an optical device that transforms any of the various polarization forms of an incident light wave into a unique polarization state at its output. In this case the determinant $T_{22} T_{11}-T_{21} T_{12}$ of the bilinear transformation vanishes and Eq. (4) becomes

$$
\chi^{\prime}=\chi_{0}=T_{22} / T_{12}=T_{21} / T_{11},
$$

where $\chi_{0}$ represents the outgoing polarization state, which is generally elliptic. The equivalent circuit analog is shown in Fig. 4(a), where $Z_{0}=\chi_{0}$. A polarizer is said to exhibit leakage if it has a small, nonzero transmission for the incident polarization state that is orthogonal with $\chi_{0}$. This condition is taken care of by replacing the short circuit of Fig. 4(a) by a small impedance $Z_{1}$ such that $\left|Z_{1}\right| \ll\left|Z_{0}\right|$ [Fig. 4(b)]. As another example, a linear dichroic plate shows nonequal but inphase transmittances along two orthogonal principal directions in its plane. In this case it can be easily shown that Eq. (4) degenerates to the simple linear transformation

$$
\chi^{\prime}=K_{\chi},
$$

where $K$ is the ratio of the amplitude transmittances along the two principal axes of dichroism. Obviously the circuit analog is an ideal transformer [Fig. 4(c)] whose turns ratio is $K^{\frac{1}{2}}$. A rotator is a device that rotates the major axis of the polarization ellipse through a fixed angle in a fixed sense for all orientations and ellipticities of the incident polarization ellipse. The cartesian Jones matrix is 


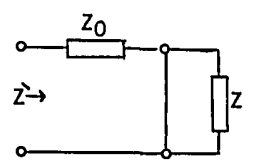

(a)

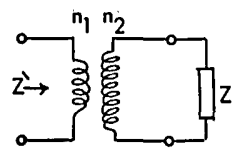

(c)

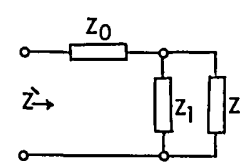

(b)

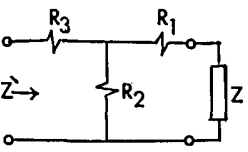

(d)
Fig. 4. The circuit analogs of some of the simple optical devices: (a) ideal polarizer, (b) imperfect polarizer with leakage

$\left|Z_{1}\right| \ll\left|Z_{0}\right|$, (c) purely dichroic plate, and (d) optical rotator.

$$
T_{r}=\left(\begin{array}{rr}
\cos \alpha & \sin \alpha \\
-\sin \alpha & \cos \alpha
\end{array}\right)
$$

which when substituted in Eq. (4) gives

$$
\chi^{\prime}=(\chi \cos \alpha-\sin \alpha) /(x \sin \alpha+\cos \alpha) .
$$

The parameters of the equivalent $T$ network are

$$
Z_{1}=-\tan (\alpha / 2), \quad Z_{2}=\csc \alpha, \quad Z_{3}=-\tan (\alpha / 2),
$$

which follows from Eqs. (27) and (30), and leads to the resistive circuit of Fig. 4(d). Note that in this case the equivalent circuit analog is not as simple as those encountered in the above two examples.

The simplicity of producing and measuring light of known polarization coupled with the fact that the optical analogs of negative impedence networks are readily realizable may make the optical simulation of electrical two-port networks of considerable practical value. However, the opposite does not appear to be as simple, namely, to simulate optical systems by electrical twoport networks as can be seen from the example of the optical rotator.

Finally, it should be noted that linear mechanoacoustic systems are described by equations similar to Eqs. (1) and (5). In this case the pair of oscillating quantities are the mechanical force (or pressure) and velocity (or displacement). Therefore these systems have their optical analogs as well as their well-known electrical analogs.

\section{Conclusions}

Analogy between optical systems and two-port electrical networks is demonstrated. In each case we deal with the transformation of a pair of oscillating quantities between input and output. The mapping of polarization by an optical system and of impedance by a two- port network is described by a bilinear transformation. For each terminal characteristic of a system of one type there is a similar characteristic for the system of the other type. For example, the two eigenpolarizations of the optical system are the analogs of the two iterative impedances of the two-port network. The loci of polarization states that preserve ellipticity or azimuth and those of impedances (or admittance) that preserve magnitude or angle are described by the same equations in the complex plane. Two-port electrical networks are synthesized whose impedance- (or admittance-) mapping properties are the same as the polarization-mapping properties of a given optical system. The opposite problem is also considered. The analogy presented in this paper is useful because (1) it unifies the methods of treating both kinds of systems and (2) it leads to the reciprocal simulation of systems of one type by systems of the other, which is of practical value.

This work was supported by the National Science Foundation.

\section{References}

1. When there is more than one incident beam or when a single incident beam generates a number of emergent beams (e.g., in the presence of a diffraction grating), the electrical analog of the optical system becomes a multiport network. There is no loss of generality, however, when a two-port network is used to represent the relation between one incident and one emergent beam.

2. W. A. Shurcliff, Polarized Light (Harvard U.P., Cambridge, 1962).

3. R. M. A. Azzam and N. M. Bashara, J. Opt. Soc. Am. 62, 222 (1972).

4. R. M. A. Azzam and N. M. Bashara, Optics Commun. 4, 203 (1971).

5. R. M. A. Azzam and N. M. Bashara, J. Opt. Soc. Am. 62, 336 (1972); Opt. Commun. 5, 5 (1972).

6. R. M. A. Azzam and N. M. Bashara, Appl. Opt. 12, January (1973).

7. Large-signal equivalent circuits are sometimes used to relate voltages and currents of the same frequency in the presence of nonlinear effects [see, e.g., M.A.H. El-Said, IEEE Trans. Circuit Theory 17, 8 (1970)]. Such circuits might simulate nonlinear optical systems if light waves of the same frequency are coherent at input and output.

8. Conventionally voltages are listed before currents in the input and output vectors.

9. V. H. Rumsey, Proc. IRE 39, 535 (1951).

10. G. A. Deschamps in Proc. Symp. Modern Network Synthesis J. Fox, Ed. (Polytechnic Press, Brooklyn, 1952).

11. E. A. Guillemin, Mathematics of Circuit Analysis, (Massachusetts Institute of Technology Press, Cambridge, 1949).

12. When the orthogonal left and right circular polarizations are used as basis states, the azimuth $\theta$ and ellipticity $e$ of the ellipse of polarization are obtained from the complex polarization variable $\chi$ [Eq. (3)] by the relations $\theta=\frac{1}{2} \operatorname{Arg}(\chi)$ and $e=(|\chi|-1) /(|\chi|+1)$. These lead to the equiazimuth-equiellipticity chart of Fig. 2 (left). 